\title{
DIFFUSION, CALCINATION, AND SINTERING PROCESS OF THE FERROELECTRIC CERAMICS
}

\author{
Open Engineering Collaboration*†
}

October 27, 2020

\begin{abstract}
In this work, we present the diffusion, calcination, and sintering processes in the production of ferroelectric ceramics.
\end{abstract}

keywords: diffusion, calcination, sintering, material sciences, ferroelectric ceramics.

\section{Introduction}

1. Science and engineering of materials investigate the production and characterization of materials, such as ferroelectric ceramics. These processes include heat treatment of calcination and sintering [1].

\section{Methods}

2. A systematic literature review was carried out in the area of materials science and engineering.

*All authors with their affiliations appear at the end of this paper.

†Corresponding author: andre@selfchargebox.com | Open Engineering Collaboration. Collaboration 


\section{Results and Discussion}

3. Ferroelectric ceramics are prepared from the mixture of precursor powders, thermal calcination process, conformation, and thermal sintering process [2].

4. Diffusion occurs in the thermal processes of calcination and sintering. Diffusion is the transport of matter (atoms) from one position to another in the crystalline network through specific defects (interstitials or gaps). The diffusion is relevant to the densification, phase formation, and final definition of the crystalline structures of the ceramic materials 3 .

5. The speed of atomic diffusion depends on the pre-exponential factor $\left(D_{0}\right)$, apparent activation energy $(Q), R$ the universal gas constant, and $T$ the temperature, as indicated by the Arrhenius equation:

6. $D=D_{0} e^{\frac{-Q}{R T}}$

7. The powder compacted (uniaxial or isostatic pressing) is fragile and with little mechanical resistance due to the large vacancy between the particles. To increase the mechanical resistance it is necessary to reduce the free surface energy of the system. Therefore, it is necessary to reduce the surface area and the particle interfaces of the powder compacted by grain growth and decrease the vacancy gradient [4].

8. The vacancy flow $(J)$ along a direction $(x)$ in a steady-state is defined by Fick's first law where $\frac{\mathrm{dC}}{\mathrm{d} x}$ is the vacancy concentration gradient:

9. $J=-D \frac{\mathrm{dC}}{\mathrm{d} x}$

10. The reduction of free energy occurs in the sintering process, which is divided into 3 stages. The first stage is when the rounding of the particles starts, forming necking and grain contours. The second stage is when grain growth begins, densification, and pore closure. Finally, 
the third stage is when the residual pores are eliminated and the grain grows [4].

\section{Final Remarks}

11. The sintering process is fundamental in obtaining the final properties of ferroelectric ceramics [6,7]. The factors that influence the sintering process are heating and cooling rate, temperature, time, particle size, particle distribution, chemical composition, additives, and atmosphere.

\section{Open Invitation}

Review, add content, and co-author this paper 8]. Join the Open Engineering Collaboration.

Send your contribution to andre@selfchargebox.com

\section{References}

[1] Carter, C. Barry, and M. Grant Norton. Ceramic materials: science and engineering. Vol. 716. New York: springer, 2007.

[2] Buchanan, R. C., ed. Ceramic materials for electronics. CRC press, 2018.

[3] Smith, W. F., Hashemi, J., and Presuel-Moreno, F. Foundations of materials science and engineering. Vol. 397. New York: McGraw-hill, 2006 .

[4] Callister, William D. Materials science and engineering an introduction. John Wiley, 2007.

[5] Aziz, S. B., "Role of dielectric constant on ion transport: Reformulated Arrhenius equation." Advances in Materials Science and Engineering., 20 Oct. 2020 https://doi.org/10.1155/2016/2527013 
[6] Bonaventura, A. L. , et al. "Properties of advanced ceramics concerning intrinsic and extrinsic factors." OSF Preprints., 13 Oct. 2020. https://doi.org/10.31219/osf.io/xt7h6

[7] Serrano, A. G., et al., "Microstructural, structural and electrical properties of bilayered BaZr0.05Ti0.95O3/Ba0.75Sr0.25TiO3 ceramics." Materials Research Bulletin., 10 Oct. 2020. https://doi.org/10.1016/j.materresbull.2016.11.016

[8] Bonaventura, A. L. , et al. "DIFFUSION, CALCINATION, AND SINTERING PROCESS IN FERROELECTRIC CERAMICS." OSF Preprints.

\section{The Open Engineering Collaboration}

André Luis Bonaventura, (lead author, andre@selfchargebox.com) $)^{1,2}$

Rangel Graudiston Aredes, (rangel.aredes@gmail.com) ${ }^{1}$

Eduardo Antonelli, (antonelli@unifesp.br) ${ }^{1}$

${ }^{1}$ Advanced Ceramic Laboratory, Science and Technology Institute, Universidade Federal de São Paulo; ${ }^{2}$ Self Charge Box, StartUp, São José dos Campos - SP - Brazil. 